\title{
Separation of chiral colloidal particles in a helical flow field $†$
}

\begin{abstract}
Maria Aristov, ${ }^{\text {ac }}$ Ralf Eichhorn ${ }^{\mathrm{b}}$ and Clemens Bechinger ${ }^{\star a c}$
Stereoisomeric molecules with opposite chirality, so called enantiomers, often vary regarding their sensory, pharmacological and toxicological properties. Such enantiomer specific effects play a central role in the development, testing and evaluation of drugs, pesticides and food related products. Accordingly, efficient techniques for separation of chiral mixtures into enantiopure compounds are of enormous practical relevance. Most current enantiomer separation methods are based on enantioselective interactions with an auxiliary substance which has to be developed and optimized for different chiral molecules in an elaborate and costly process. Here, we experimentally demonstrate the separation of micron sized chiral particles in a helical fluid flow which is created inside a microfluidic device patterned with slanted grooves. We observe that the retention time of particles in a helical flow field strongly depends on their chirality which leads to an effective chiral separation within the channel. Our experimental results are confirmed by numerical calculations which demonstrate how the coupling of rotational and translational degrees of freedom leads to differences in the trajectories of particles with opposite chirality. Since our separation mechanism does not rely on material specific interactions, this offers considerable advantages over existing methods. We expect that our approach can be also applied at nanometre length scales by using channels with smaller diameters and with an optimized geometry.
\end{abstract}

\section{Introduction}

Organic and inorganic molecules often have a chiral structure or handedness, i.e. the molecule and its mirror image are not superimposable. Despite an identical chemical composition, the pharmacological and toxicological effects caused by left- and right-handed enantiomers are not always the same. ${ }^{14}$ The variations can be subtle as e.g. in the case of limonene whose enantiomers have either an orange or lemon aroma. ${ }^{5}$ However, dramatic differences can occur with thalidomide being probably the most prominent example: ${ }^{6}$ while one chirality can treat

${ }^{a} 2^{\text {nd }}$ Physical Institute, Physics Department, University of Stuttgart, Pfaffenwaldring 57, 70569 Stuttgart, Germany. E mail: c.bechinger@physik.uni stuttgart.de; Tel: +49 71168565218

${ }^{b}$ Nordita, Royal Institute of Technology and Stockholm University, Roslagstullsbacken 23, SE 10691 Stockholm, Sweden

${ }^{c}$ Max Planck Institute for Intelligent Systems, Heisenbergstrasse 3, 70569 Stuttgart, Germany morning sickness, the other was later found to be responsible for physical birth defects. Since chemical synthesis of stereoisomeric substances typically yields both chiralities in equal amounts (racemic mixture), pharmacological tests must be performed for each single enantiomer and, if necessary, only enantiopure products are approved for medical use. ${ }^{7}$ This requires efficient and versatile methods for the separation of chiral molecules. Standard enantiomer separation techniques typically make use of chirality specific chemical interactions which allow separation of racemic mixtures by e.g., chromatographic methods ${ }^{8}$ or capillary electrophoresis. ${ }^{9}$ To avoid the development of chemical agents matching specific enantiomers, hydrodynamic shear or vortex flows, ${ }^{10}{ }^{16}$ lattice potentials, ${ }^{17,18}$ and polymer networks with imprinted chiral texture ${ }^{\mathbf{1 9}, 20}$ have been proposed as alternative separation media. Although hydrodynamic effects depending on the particle's chirality have been experimentally studied for different Reynolds numbers ${ }^{21,22}$ and recently observed even at micrometre length scales, ${ }^{14}$ so far the separation of racemic particle mixtures has not been demonstrated.

Here, we experimentally and numerically demonstrate the principle of chiral separation in a helical flow field at mesoscopic length scales. We use micron-sized chiral particles whose motion can - in contrast to chiral molecules - be individually followed by video microscopy which allows us to reveal how the coupling of rotational and translational degrees of freedom leads to chirality dependent retention times and thus to chiral separation. 


\section{Experimental system}

Three-dimensional particles with right ( $R$ for rectus) and left handed ( $S$ for sinister) chiralities and dimensions of $6 \times 6 \times$ $6 \mu \mathrm{m}^{3}$ are made from photoresist SU-8 (MicroChem) by photolithography according to ref. 23 and labelled with green $(R, 0.04$ $\mathrm{mg} \mathrm{ml} \mathrm{m}^{-1}$ Nile Red, Invitrogen) and blue ( $S, 0.1 \mathrm{mg} \mathrm{ml}^{-1} 2.6$-ANS, Invitrogen) fluorescent dyes ${ }^{24}$ (ESI Movie1†) (Fig. 1A and B). Microfluidic channels as shown in Fig. 1C with rectangular cross-sections and periodically arranged grooves at the top are created from thin layers of an elastomer (polydimethylsiloxane, PDMS) which are attached to microscope slides. ${ }^{25}$ Application of a hydrostatic pressure of $\Delta P=5 \times 10^{3}$ Pa to such devices by connecting the channel's inlet and outlet to reservoirs with different water levels leads to a helical flow field whose circulating direction is determined by the orientation of the ridges relative to the pressure drop. ${ }^{26}$ Typical particle concentrations for continuous and pulsed measurements ranged between 10 and $10^{5}$ particles per $\mu$ l. The particle motion projected onto the $x y$-plane was imaged using an inverted fluorescent microscope coupled to a video camera. Particle numbers were obtained by digital video microscopy at a frame rate of $12 \mathrm{fps}$ and an exposure time of $70 \mathrm{~ms}$. At a typical flow velocity of $1 \mathrm{~mm} \mathrm{~s}^{-1}$, particle images appear as green and blue streaks with approx. $10 \mu \mathrm{m}$ of length (ESI Movie2 $\dagger$ ). From the number of streaks the $R: S$ particle ratio and the particle number averaged over channel segments of $1 \mathrm{~mm}$ are obtained.

\section{Theoretical model and numerical simulations}

The dynamics of chiral particles is modelled by the overdamped equations of motion

$$
\left(\begin{array}{c}
\dot{\vec{r}} \\
\dot{\vec{\varphi}}
\end{array}\right)=\left(\begin{array}{ll}
\mu^{\mathrm{tt}} & \mu^{\mathrm{tr}} \\
\mu^{\mathrm{rt}} & \mu^{\mathrm{rr}}
\end{array}\right)\left(\begin{array}{c}
\vec{F} \\
\vec{T}
\end{array}\right)+\left(\begin{array}{c}
\vec{\xi}_{r}(t) \\
\vec{\xi}_{\varphi}(t)
\end{array}\right)
$$

Here, $\vec{r}$ and $\vec{\varphi}$ represent the particle's translational and rotational degrees of freedom and $\mu=\left(\begin{array}{ll}\mu^{\mathrm{tt}} & \mu^{\mathrm{tr}} \\ \mu^{\mathrm{rt}} & \mu^{\mathrm{rr}}\end{array}\right)$ is the $6 \times 6$ mobility tensor which describes the coupling between particle translation and rotation via tensor components $\mu^{\text {tr }}$ and $\mu^{\text {rt }} \cdot \vec{F}$ and $\vec{T}$ are the hydrodynamic friction forces and torques imposed on the particle by the flow field inside the patterned channel (see ESI $\dagger$ ). The last term in eqn (1) corresponds to Gaussian distributed white noise $\xi(t)=\left(\begin{array}{l}\vec{\xi}_{r}(t) \\ \vec{\xi}_{\varphi}(t)\end{array}\right)$ with $\left\langle\xi_{i}(t) \xi_{j}(s)\right\rangle=$ $2 D_{i j} \delta(t \quad s)(i, j \in\{1,2, \ldots, 6\})$ and the diffusion tensor $D_{i j}$ which obeys the Einstein relationship $D_{i j}=\mu_{i j} k T$ with $k T$ being the thermal energy. To allow for comparison with experimental data, eqn (1) is sampled over different initial conditions and realizations of the thermal noise. Since the mean particle distance in our experiments is about $40 \mu \mathrm{m}$, direct particle interactions have been neglected in the simulations. Chiral particles are modelled by seven rigidly connected spherical beads of $1 \mu \mathrm{m}$ radius which closely resembles the dimensions of the particles used in the experiments (Fig. 1A). ${ }^{27}$ Based on the friction tensors for the hydrodynamic interactions between any pair of such beads, the mobility tensor $\mu=\left(\begin{array}{ll}\mu^{\mathrm{tt}} & \mu^{\mathrm{tr}} \\ \mu^{\mathrm{rt}} & \mu^{\mathrm{rr}}\end{array}\right)$ was determined (see ESI $\dagger$ ). For a single spherical bead, eqn (1) reduces to a simple equation for $\vec{r}$ with scalar $\mu^{\text {tt }}$, because of the irrelevant rotational degrees of freedom $\left(\mu^{\mathrm{tr}}=\mu^{\mathrm{rt}}=\mu^{\mathrm{rr}}=0\right)$.

For the numerical simulations of the particle motion, first the fluid flow (in the absence of any particles) is computed in a part of the structured channel consisting of five periods (in the $x$ direction) by solving the Navier-Stokes equation for vanishing Reynolds number ${ }^{28,29}$ with no-slip boundary conditions at fluidsolid interfaces and for a prescribed pressure drop over the channel length using COMSOL. Then, the resulting solution for the flow velocity and vorticity in the central basic period is periodically continued and used as the "external" flow field to calculate $\vec{F}$ and $\vec{T}$ when numerically integrating the equations of motion eqn (1). Integration is performed by using the Euler algorithm (time-step $1 \mu$ s or smaller), and collisions of the particles with the (hard) channel walls are treated by the so-called rejection scheme. ${ }^{30}$

\section{Results and discussion}

For the experimental demonstration of chiral separation, we injected a single pulse of a chiral particle mixture into a channel with a right handed flow circulation (Fig. 1C). A spatially sharp defined pulse of high particle concentration was created via
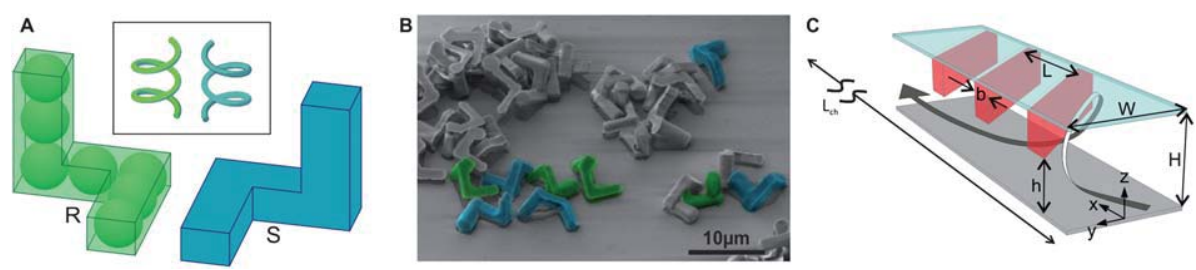

Fig. 1 Fabrication of chiral microparticles and microfluidic channels with a helical flow field. (A) Schematic illustration of particles of opposite chirality $R$ (green/light grey) and $S$ (blue/dark grey) and their description by seven spherical beads in the numerical simulations. For the definition of the particle chirality we compare them with right and left handed spirals (inset). (B) SEM micrograph of chiral particles with some particles artificially coloured corresponding to their chirality. (C) Microfluidic channels made from PDMS (light blue and red; side walls omitted to enhance visibility) deposited on a microscope slide with width $W=150 \mu \mathrm{m}$ and height $H=115 \mu \mathrm{m}$. PDMS is periodically patterned with obliquely $\left(45^{\circ}\right.$ ) oriented ridges (red/dark grey) with height $H \quad h=50 \mu \mathrm{m}$, width $b=40 \mu \mathrm{m}$ and centre centre distance $L=146 \mu \mathrm{m}$. Application of a pressure gradient along the channel leads to a right handed helical flow field (arrow). The total length of the channel, which consists of 15 straight segments of $65 \mathrm{~mm}$ length connected by bends of $180^{\circ}$, is $L_{\mathrm{ch}}=1 \mathrm{~m}$. 

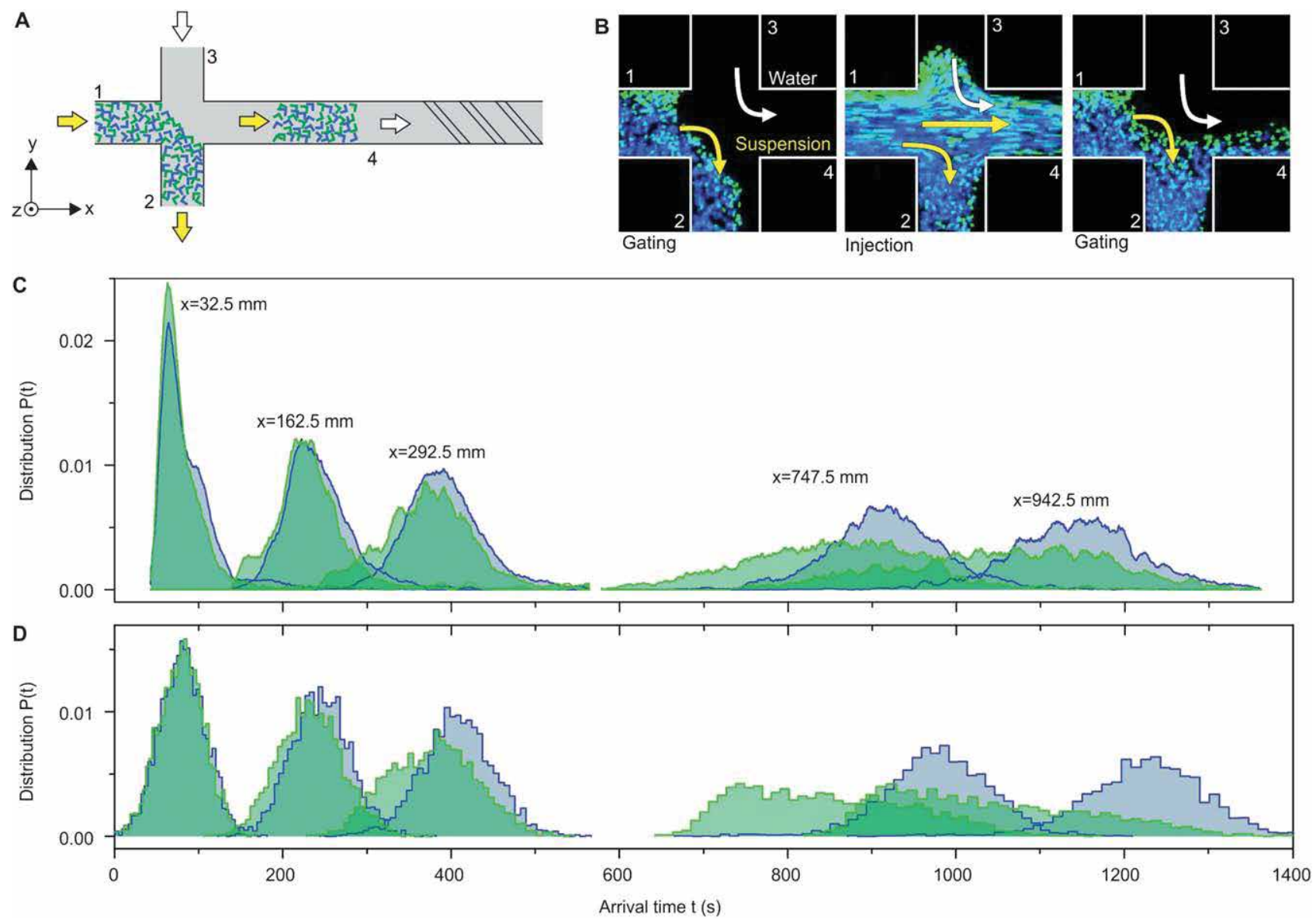

Fig. 2 Chiral separation in a right handed helical flow field. (A) Principle of hydrodynamic gating for insertion of a spatially confined volume of a chiral mixture into a microchannel. During the injection process, the pressures at the inlets 1, 3 and the outlets 2, 4 are varied as follows: first the pressures at the inlets 1, 3 and the outlets 2 , 4 are adjusted to $p_{1}=p_{3}$ and $p_{2}=p_{4}$ with $p_{2}<p_{1}$. This leads to two flows with constant rates $Q_{12}=Q_{34}$. During injection, $p_{1}$ is increased for about one second and then returned to its original value. (B) Snapshots of the measured fluorescent images during injection of a single pulse of a chiral mixture. (C) Measured arrival time distributions of a total number of about $5600 R$ (green/light grey) and S (blue/dark grey) particles at different channel positions $x$ relative to the injection point. (D) Corresponding data from numerical simulations obtained from 1000 realizations.

hydrodynamic gating ${ }^{31}$ which is achieved by applying a timedependent pressure sequence to the cross-shaped inlet of the channel (Fig. 2A and B). The relative particle mixture and the initial total concentration of the pulse were determined from single particle tracking to be $R: S=47: 53$ and $10^{5}$ per $\mu$ l corresponding to a volume fraction of $0.56 \%$. Fig. $2 \mathrm{C}$ shows the measured arrival time distributions of $R$ and $S$ particles at different channel positions $x$ for a pressure gradient of $\Delta P \cong$ $5 \times 10^{3}$ Pa which leads to a mean particle velocity of approx. 0.85 $\mathrm{mm} \mathrm{s}^{-1}$. At $x=32.5 \mathrm{~mm}$, both distributions are rather similar which suggests that we have inserted a uniform particle mixture. With increasing $x$, we observe a broadening and a relative shift of the arrival time distributions of $R$ and $S$ particles. Obviously, right-handed $(R)$ particles propagate in a righthanded flow field faster than $S$ particles. At $x=942.5 \mathrm{~mm}$, the fastest $R$ particles arrive almost $200 \mathrm{~s}$ before the $S$ ones. From Fig. 2C, we estimate that about $25 \%$ of the injected $R$ particles can be extracted without $S$ impurities.

For a quantitative understanding of why the velocity of enantiomers in a helical liquid flow depends on the particle's chirality, we numerically computed particle trajectories from the corresponding overdamped equations of motion eqn (1). To compare the numerical simulations with experimental data, we first studied the motion of spherical tracer particles with $1 \mu \mathrm{m}$ diameter in a patterned microfluidic channel with a righthanded helical liquid flow. Typical trajectories projected onto the $x y$-plane are plotted in Fig. $3 \mathrm{~A}$ as symbols (experiment) and coloured solid lines (simulations) and show good agreement. As a result of the no-slip boundary conditions at the channel surfaces, the fluid flow - and thus the particle velocity - becomes largely reduced at these regions. This is indicated by the shorter distance of the measured particle's positions which were taken at time intervals of $t=21 \mathrm{~ms}$. In contrast, particles traveling close to the channel axis have more than the double speed.

Since we obtain the full three-dimensional trajectory from the numerical computations, a more detailed analysis of the particle's motion is possible. We superimposed the particle's $y z$ location at eight periodic $x$ positions $x_{i+1}=x_{i}+L$ (shown by the vertical dashed lines in Fig. 3A). Such Poincaré maps provide a convenient representation of complex particle dynamics since they visualize typical particle orbits. Also, they illustrate the lateral particle distribution in the course of its transit through 
A

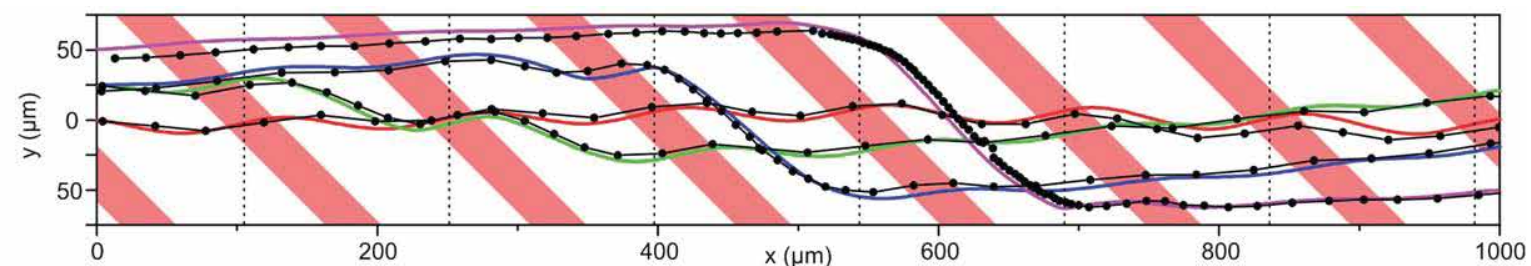

B

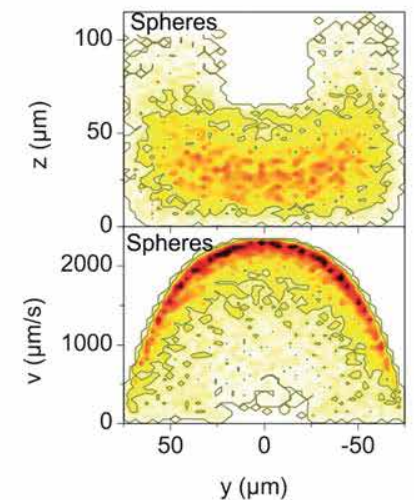

H

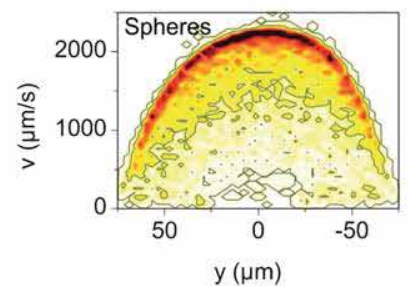

C
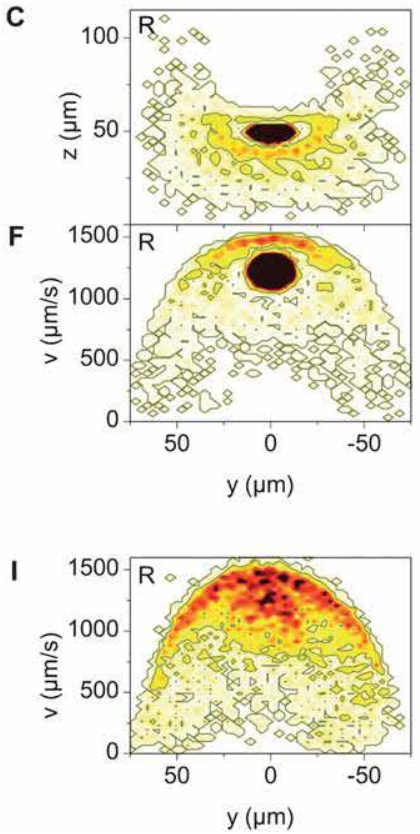

D
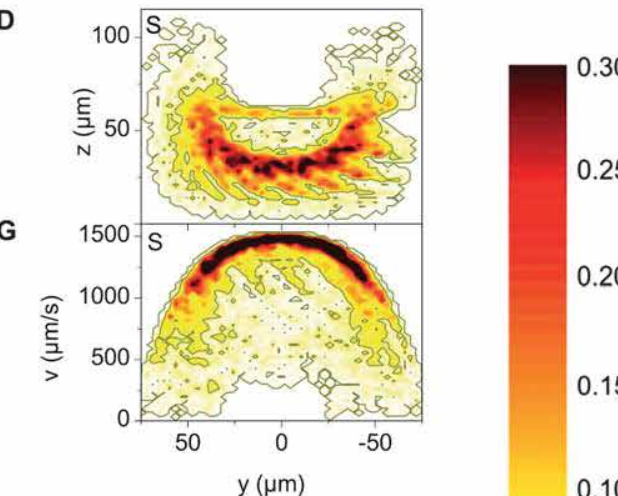

0.30

0.25

0.15

0.10

J

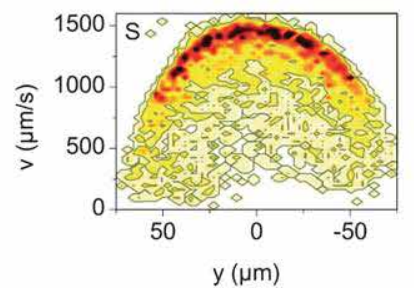

0.05

0.00

Fig. 3 Particle trajectories and Poincaré maps. (A) Measured (symbols) and simulated (solid lines) trajectories of spherical particles with $1 \mu \mathrm{m}$ diameter flowing from left to right through a patterned channel with a right handed helical flow field. The slanted red areas correspond to the protruding ridges at the top wall. Normalized density plots of Poincaré maps of the particle position within the channel cross section obtained from 1500 simulated trajectories each for (B) spheres, (C) $R$ particles and (D) $S$ particles. Due to the minimization of viscous forces, the spatial distribution of particles strongly depends on their shape and chirality. The filament like structures at the channel walls in (D) are signatures of deterministic particle trajectories (attractors) and arise from the coupling between translational and rotational degrees of freedom. Due to imperfections of the channel structure and a finite particle polydispersity, this effect is less pronounced in experiments. Corresponding Poincaré maps of the particle velocity along the $x$ direction and their $y$ position obtained from simulations and experiments for (E and $\mathrm{H}$ ) spheres, ( $\mathrm{F}$ and $\mathrm{I}) \mathrm{R}$ particles, and ( $G$ and J) S particles.

the channel. For spherical tracers the Poincare map shows a rather uniform and uncorrelated particle distribution within the geometrically accessible channel cross-section. This is consistent with the fact that fluids can be efficiently mixed in such patterned microchannels. ${ }^{25}$ For chiral particles a different behaviour is observed. Most trajectories of $R$ particles are confined to a rather narrow region close to the central channel axis (Fig. 3C). The Poincare map for $S$ particles shows the opposite behaviour, i.e. a reduction of data points at the channel centre. Obviously, $S$ particles prefer to travel closer to the channel walls (Fig. 3D). To understand why the spatial distributions of $R$ and $S$ particles are different, one has to recall that particles always prefer those positions where their frictional losses with the surrounding liquid become minimal. Therefore, the velocity difference between the particle's surface and the fluid is minimized. For $R$ particles in a right-hand flow, this is achieved by moving close to the fluid vortex centre. In contrast, the viscous friction for $S$ particles becomes maximum in this region because segments of the particle's surface move even opposite to the fluid flow.
Owing to the inhomogeneous velocity profile within the channel, any difference in the spatial particle distribution must lead to variations in the mean particle velocity. This is shown in Fig. 3E-J where we compare simulated (E-G) and experimentally $(\mathrm{H}-\mathrm{J})$ measured Poincare maps of the particle velocity along the $x$ direction versus their $y$ coordinate. For spherical tracers, the numerical simulations predict a parabolic velocity profile which is also observed in the experimental data (Fig. 3E and $\mathrm{H}$ ). In addition to such parabolic envelopes, pronounced chirality dependent features are present in the corresponding plots of $R$ and $S$ particles: for $R$ particles, the simulations and the experimental data show a strong accumulation of data points around the channel centre at $y=0$ (Fig. $3 \mathrm{~F}$ and I). Due to the high flow velocity in that region, $R$ particles travel with around 1050-1400 $\mu \mathrm{m} \mathrm{s}^{-1}$ (Fig. 3F). For $S$ particles, no such accumulation at the channel centre is observed (Fig. $3 \mathrm{G}$ and J). As a consequence, they are preferably localized closer to the channel walls where their velocity is small. From the numerical simulations we find that $R$ and $S$ particles are transported with an average velocity of $1200 \mu \mathrm{m} \mathrm{s}^{-1}$ and $1060 \mu \mathrm{m} \mathrm{s}^{-1}$, respectively. This difference 

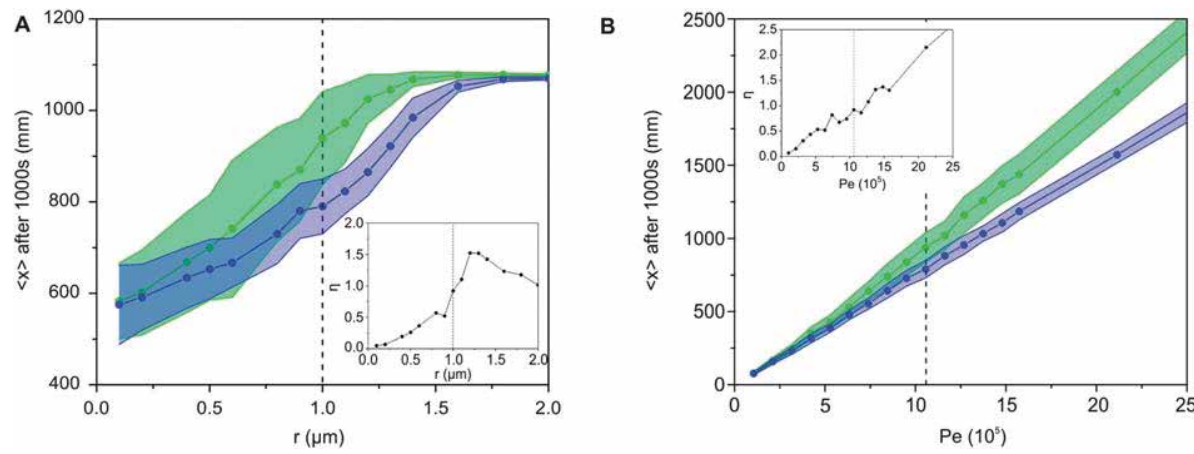

Fig. 4 Separation efficiency obtained by numerical simulations. (A) Average position of the injected particle pulse $\langle x\rangle$ after $1000 \mathrm{~s}$ in a right handed flow field as in Fig. 2D for $R$ (green/light grey) and $S$ (blue/dark grey) particles as a function of the bead radius of the model chiral particle (see Fig. $1 A$ ). Green and blue areas correspond to the standard deviations $\sigma_{R}$ and $\sigma_{S}$. The inset shows the particle separation efficiency $\eta=\left|\langle x\rangle_{R} \quad\langle x\rangle_{S}\right| /\left(\sigma_{R}+\sigma_{S}\right)$. Vertical dashed lines correspond to experimental conditions. (B) Dependence of $\langle x\rangle$ on the Péclet number Pe $=L v / D$ with $L$ the spatial period of the patterned channel, $v$ the average fluid velocity in the $x$ direction obtained from the mean value of the computed chiral flow field and $D$ the particle translational diffusion coefficient. The inset shows the particle separation efficiency vs. the Péclet number. Vertical dashed lines correspond to experimental conditions, i.e. $L=146 \mu \mathrm{m}, D=0.08 \mu \mathrm{m}^{2} \mathrm{~s}{ }^{1}, v=580 \mu \mathrm{m} \mathrm{s}^{1}$.

allows for an efficient particle separation as shown in Fig. 2D, where we plot the arrival time distributions from simulated trajectories which show a good agreement with the experimental data in Fig. 2C. In addition to the position of the peaks, also the chirality dependent dispersion of the arrival time distributions is well reproduced. Due to the higher velocity variations observed for $R$ particles (Fig. $3 \mathrm{~F}$ and I), the corresponding distributions are much wider than those of $S$ particles (Fig. 3G and J). Both the shift between the arrival time maxima and the chirality dependent dispersion lead to an efficient separation mechanism. Since the distributions of $R$ and $S$ particles at $x=0$ are experimentally not accessible, in the simulations we assumed Gaussian distributions with a standard deviation of $25 \mathrm{~s}$ each; under these conditions best agreement with the measured arrival time distributions at $x=$ $32.5 \mathrm{~mm}$ is obtained. The simulations predict an even higher separation efficiency compared to our experiments. We attribute these deviations to imperfections in the channel structure and a finite polydispersity in shape and size of the fabricated chiral particles. The overall good agreement, however, suggests that the separation of chiral particles with helical flow fields is robust.

We also studied the separation efficiency as a function of the particle size, i.e. the bead radius $r$ as defined in Fig. 1A. This is shown in Fig. 4A where we plot the average position $\langle x\rangle$ covered by $R$ and $S$ particles $v s . r$ for the same conditions used in Fig. 2D. The position $\langle x\rangle$ increases with the bead radius until above $r>1.5 \mu \mathrm{m}$ a saturation is observed, because then both particle types preferably move close to the channel axis where the flow velocity becomes maximal. We find that the particle separation efficiency for our channel geometry becomes maximized around $r=1.2 \mu \mathrm{m}$ which is close to the particle dimensions used in our experiments (inset in Fig. 4A). Fig. 4B shows the same data plotted $v s$. the Péclet number. Viscous forces are proportional to the velocity, therefore the mean particle position and the chiral separation efficiency increase monotonically with the increasing Péclet number. By simple scaling arguments one can estimate from Fig. 4 the lower particle size limit which can be separated with the channel geometry considered here. Assuming a Péclet number $\mathrm{Pe}=10^{6}$ required for efficient separation and currently technically achievable pressure heads of $2 \times 10^{8} \mathrm{~Pa}$ (Microfluidics Corp., Newton, MA), ${ }^{14}$ it can be estimated from the Hagen-Poiseuille equation that particles of about $120 \mathrm{~nm}$ can be separated in a channel with $3.2 \mu \mathrm{m}$ crosssection and $3 \mathrm{~cm}$ length. We expect that this size limit can be further reduced by optimizing the geometry of the channels ${ }^{32}$ where chiral separation is achieved. In contrast to our devices where only the upper wall was patterned, e.g. channels with circular cross-sections which are patterned on the entire inner surface $^{33}$ should largely pronounce the vorticity of the fluid flow and thus enhance the separation efficiency for particles in the nanometre range.

\section{Acknowledgements}

We acknowledge the assistance of Stephen Riedel during the imaging of chiral colloidal particles with the electron microscope and Hans Behringer for his contributions during the development of the numerical code used for the simulations.

\section{References}

1 D. Burke and D. J. Henderson, Br. J. Anaesth., 2002, 88, 563576.

2 T. J. Maher and D. A. Johnson, Drug Dev. Res., 1991, 24, 149156.

3 B. Kasprzyk-Hordern, Chem. Soc. Rev., 2010, 39, 4466-4503.

4 C. Bicchi, C. Cagliero and P. Rubiolo, Flavour Fragrance J., 2011, 26, 321-325.

5 M. Laska and P. Teubner, Chem. Senses, 1999, 24, 161-170.

6 J. Botting, Drug News Perspect., 2002, 15, 604-611.

7 Chirality, 1992, 4, 338-340.

8 G. Gübitz and M. G. Schmid, Biopharm. Drug Dispos., 2001, 22, 291-336.

9 B. Chankvetadze, J. Chromatogr., A, 2007, 1168, 45-70.

10 M. Makino and M. Doi, Phys. Fluids, 2005, 17, 103605. 
11 R. Eichhorn, Phys. Rev. Lett., 2010, 105, 034502.

12 S. Meinhardt, J. Smiatek, R. Eichhorn and F. Schmid, Phys. Rev. Lett., 2012, 108, 214504.

13 C. I. Mendoza, C. M. Marques and F. Thalmann, Phys. Rev. E: Stat., Nonlinear, Soft Matter Phys., 2010, 82, 060401 (R).

14 Marcos, H. C. Fu, T. R. Powers and R. Stocker, Phys. Rev. Lett., 2009, 102, 158103.

15 M. Kostur, M. Schindler, P. Talkner and P. Hänggi, Phys. Rev. Lett., 2006, 96, 014502.

16 P. Talkner, G.-L. Ingold and P. Hänggi, New J. Phys., 2012, 14, 073006.

17 D. Speer, R. Eichhorn and P. Reimann, Phys. Rev. Lett., 2010, 105, 090602.

18 L. Bogunovic, M. Fliedner, R. Eichhorn, S. Wegener, J. Regtmeier, D. Anselmetti and P. Reimann, Phys. Rev. Lett., 2012, 109, 100603.

19 Y. Mao and M. Warner, Phys. Rev. Lett., 2001, 86, 5309.

20 S. Courty, A. R. Tajbakhsh and E. M. Terentjev, Phys. Rev. E: Stat., Nonlinear, Soft Matter Phys., 2006, 73, 011803.

21 P. Chen and C.-H. Chao, Phys. Fluids, 2007, 19, 017108.

22 M. Makino, L. Arai and M. Doi, J. Phys. Soc. Jpn., 2008, 77, 064404 .
23 S. Badaire, C. Cottin-Bizonne, J. W. Woody, A. Yang and A. D. Stroock, J. Am. Chem. Soc., 2007, 129, 40-41.

24 C. J. Hernandez and T. G. Mason, J. Phys. Chem. C, 2007, 111, 4477-4480.

25 A. D. Stroock, S. K. W. Dertinger, A. Ajdari, I. Mezic, H. A. Stone and G. M. Whitesides, Science, 2002, 295, 647.

26 H. Wang, P. Iovenitti, E. Harvey and S. Masood, J. Micromech. Microeng., 2003, 13, 801-808.

27 B. Carrasco and J. García de la Torre, J. Chem. Phys., 1999, 111, 4817-4826.

28 J. Happel and H. Brenner, Low Reynolds Number Hydrodynamics, Martinus Nijhoff Publishers, The Hague, 2nd edn, 1983.

29 H. Brenner, J. Colloid Interface Sci., 1967, 23, 407-436.

$30 \mathrm{H}$. Behringer and R. Eichhorn, Phys. Rev. E: Stat., Nonlinear, Soft Matter Phys., 2011, 83, 065701(R).

31 P. Chen, X. Feng, J. Sun, Y. Wang, W. Du and B.-F. Liu, Lab Chip, 2010, 10, 1472-1475.

32 H. Sato, S. Ito, K. Tajima, N. Orimoto and S. Shoji, Sens. Actuators, A, 2005, 119, 365-371.

33 C. Jeon, H. Jeong and Y. Jung, Phys. Rev. E: Stat., Nonlinear, Soft Matter Phys., 2011, 83, 056324. 